\title{
Sub-femtosecond synchronization of microwave oscillators with mode-locked Er-fiber lasers
}

\author{
Kwangyun Jung and Jungwon Kim* \\ KAIST Institute for Optical Science and Technology and School of Mechanical, Aerospace and Systems Engineering, \\ Korea Advanced Institute of Science and Technology (KAIST), Daejeon 305-701, South Korea \\ *Corresponding author: jungwon.kim@kaist.ac.kr
}

Received Month X, XXXX; revised Month X, XXXX; accepted Month X,

XXXX; posted Month X, XXXX (Doc. ID XXXXX); published Month X, XXXX

\begin{abstract}
We synchronize a 8.06-GHz microwave signal from a voltage-controlled oscillator with an optical pulse train from a 77.5$\mathrm{MHz}$ mode-locked Er-fiber laser using a fiber-based optical-microwave phase detector. The residual phase noise between the optical pulse train and the synchronized microwave signal is $-133 \mathrm{dBc} / \mathrm{Hz}(-154 \mathrm{dBc} / \mathrm{Hz})$ at $1 \mathrm{~Hz}(5 \mathrm{kHz})$ offset frequency, which results in 838 as integrated rms timing jitter [1 Hz-1 MHz]. The long-term residual phase drift is 847 as (rms) measured over 2 hours, which reaches $4 \times 10^{-19}$ fractional frequency instability at $1800 \mathrm{~s}$ averaging time. This method has a potential to provide both sub-fs-level short-term phase noise and long-term phase stability in microwave extraction from mode-locked fiber lasers. (C) 2012 Optical Society of America

OCIS Codes: $320.7090,350.4010,060.5625,320.7160,120.3940$
\end{abstract}

Ultralow phase noise microwave sources are important for many scientific and engineering applications such as time-frequency metrology and standards, synchronization of large-scale scientific facilities (such as free-electron lasers), radars, networks, communication, and signal measurement instruments. Various types of ultralow noise microwave sources have been developed for the last 30 years or so, which culminated in state-of-the-art commercial microwave sources such as sapphire-loaded cavity oscillators (SLCOs) [1] and optoelectronic oscillators (OEOs) [2]. Recently, photonic generation of microwave signals from stabilized femtosecond mode-locked lasers has been an active field of research due to the ultralow noise properties of cavity-stabilized cw lasers and modelocked lasers [3-8]. It has been even shown that solid-state or fiber mode-locked lasers themselves can generate sub100-as level timing jitter optical pulse trains [9-11].

Although the optical pulse trains can carry ultralow timing jitter below a femtosecond, the transfer of timing stability from the optical domain to the electronic domain is highly nontrivial. When direct photodetection is used, excess phase noise is added in the optical-to-electronic (O/E) conversion process due to nonlinearity, saturation, temperature drift, and amplitude-to-phase conversion in photodiodes [12-14]. In order to address this issue, several approaches have been recently demonstrated. The repetition rate scaling by external interferometers has greatly improved the short-term residual phase noise well below $-160 \mathrm{dBc} / \mathrm{Hz}$ level $[15,16]$. On the other hand, to address the long-term phase stability, optical feedback control has enabled sub-fs relative timing drift [5,17].

Although the short-term phase noise or the long-term phase stability has been greatly improved by various methods [5, 8, 15-17], it would be desirable to have a method that can scale down the residual short-term jitter and long-term drift simultaneously. For this task, phase detection method based on phase error-dependent intensity imbalance between the two outputs from the Sagnac loop interferometer [18] can be used. However, the practical applicability and achievable performance were limited by alignment sensitivity, resulting in only $60 \mathrm{fs}$ precision synchronization so far [18]. In this Letter we extend this idea to a robust fiber-based optical-microwave phase detector that enables both ultralow residual shortterm $(<1 \mathrm{~s})$ phase noise and long-term $(>1 \mathrm{~h})$ phase drift below a femtosecond level. An 8.06-GHz microwave signal from a voltage-controlled oscillator ( $\mathrm{VCO}$ ) is synchronized with a $77.5-\mathrm{MHz}$ femtosecond mode-locked Er-fiber laser with out-of-loop residual phase noise of $-133 \mathrm{dBc} / \mathrm{Hz}(-154$ $\mathrm{dBc} / \mathrm{Hz})$ at $1 \mathrm{~Hz}(5 \mathrm{kHz})$ offset frequency, which results in 838 as integrated rms timing jitter $[1 \mathrm{~Hz}-1 \mathrm{MHz}]$. The long-term residual out-of-loop rms phase drift is measured to be 847 as (rms) over 2 hours.

For long-term stable, high-precision synchronization between microwave VCOs and mode-locked Er-fiber lasers, we detect and compensate for the phase error between them in the optical domain using a fiber-based optical-microwave phase detector. Figure 1 shows the schematic of the fiber-based optical-microwave phase detector. It is based on the phase detection using the phase error-dependent intensity imbalance between the two outputs from the Sagnac-loop interferometer $\left(P_{1}=P_{i n} \cos ^{2}(\Delta \Phi / 2)\right.$ and $P_{2}=P_{i n} \sin ^{2}(\Delta \Phi / 2)$, where $P_{i n}$ is Sagnac-loop input optical power and $\Delta \Phi$ is the phase difference between counter-propagating pulses in the loop). The polarization-maintaining (PM) fiber loop is built with a 50:50 coupler and a unidirectional high-speed $\mathrm{LiNbO}_{3}$ phase modulator. When a microwave signal with a frequency of integer-multiple to the laser repetition rate is applied to the phase modulator, the phase of optical pulses is modulated according to the temporal position between the optical pulses and the driving microwave signals. The intensity balancing between the two outputs from the Sagnac-loop is obtained when the phase difference $\Delta \Phi$ is set to $\pi / 2$. This necessitates a nonreciprocal quarter-wave bias between counterpropagating pulses in the fiber loop in order to lock the optical pulse train at the zero-crossings of the microwave signal. For this, we employ a nonreciprocal quarter-wave bias unit with two Faraday rotators and a quarter-wave 
plate in the fiber loop [19]. As we use a unidirectional phase modulator, the power difference between the two Sagnac-loop outputs is proportional to the phase error between the optical pulse train and the microwave signal $\left(\theta_{e}\right.$ in Fig. 1). When detected by a balanced photodetector, the output voltage signal can be used for precise opticalmicrowave phase detection. The phase error detection sensitivity is expressed as $K_{d}=G R P_{\text {avg }} \phi_{o}$ [V/rad], where $G$ is transimpedance gain of the balanced photodetector, $R$ is the responsivity of photodiodes; $P_{\text {avg }}$ is the average optical power at the two Sagnac-loop output ports; $\phi_{o}$ is the modulation depth of the phase modulator.

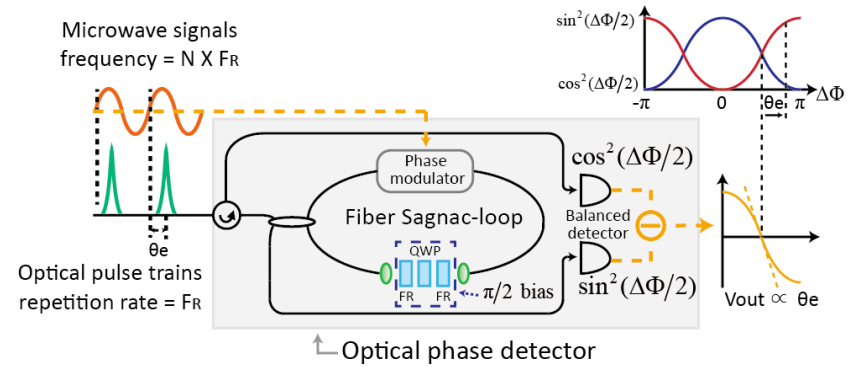

Fig. 1. Fiber-based optical-microwave phase detector.

Figure 2 shows the schematic of the optical-microwave synchronization and the out-of-loop phase measurement setup. Using the fiber-loop-based optical phase detector and a phase-locked loop (PLL) scheme, an $8.06-\mathrm{GHz}$ (the $104^{\text {th }}$ harmonic of the pulse repetition rate) microwave signal from a VCO (Hittite HMC-C200) is synchronized with a $77.5-\mathrm{MHz}$ optical pulse train generated from a home-built, low-jitter mode-locked Er-fiber laser [9]. For the out-of-loop residual phase noise and drift measurement, we built a second optical phase detector. After EDFA and 50:50 coupler, $\sim 40 \mathrm{~mW}$ optical power is applied to each optical phase detector. When the VCO is locked to the optical pulse train, $\sim 3.5 \mathrm{~mW}$ optical power is detected at each photodiode in the balanced photodetector due to insertion losses of the phase modulator and the bias unit in the fiber loop. Microwave power of $+14 \mathrm{dBm}$ from the VCO is amplified to $+21 \mathrm{dBm}$, and $+17 \mathrm{dBm}$ is applied to each phase modulator. When these optical and microwave power levels are used, the amplitude-to-phase coefficient of the optical phase detector (at $8.06 \mathrm{GHz}$ carrier $)$ is measured to be $0.06 \mathrm{rad} /\left(\Delta \mathrm{P} / \mathrm{P}_{0}\right)$ and 0.3 $\mathrm{rad} /\left(\Delta \mathrm{P} / \mathrm{P}_{0}\right)$ at $5 \mathrm{kHz}$ and $4 \mathrm{~Hz}$ offset frequency, respectively. To attain enough phase margin for the PLL, a combination of lead compensator and proportionalintegral (PI) controller is employed. The output of the outof-loop optical phase detector is applied to the $\mathrm{RF}$ spectrum analyzer and the data acquisition board for measuring residual phase noise and drift, respectively.

Figure 3(a) shows the single-sideband (SSB) phase noise measurement results at $8.06 \mathrm{GHz}$ carrier frequency: curve (i) shows the phase noise of the free-running VCO and curve (ii) shows the out-of-loop residual phase noise of the locked VCO. Curve (ii) is subtracted by $3 \mathrm{~dB}$ within the locking bandwidth $(<\sim 100 \mathrm{kHz})$ from the measured data in order to represent the noise of a single system because the measured data is limited by the same additive phase noise in phase detectors within the locking

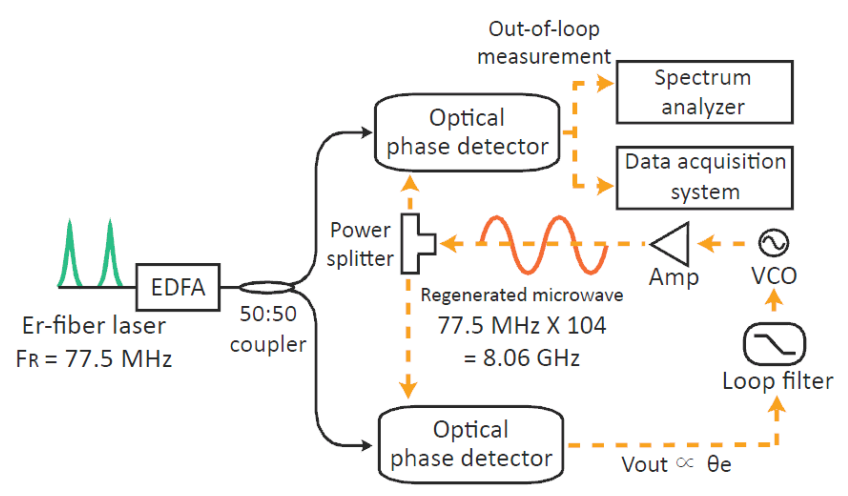

Fig. 2. Optical-microwave synchronization and out-of-loop phase noise/drift measurement set-up.

bandwidth. The residual phase noise is $-133 \mathrm{dBc} / \mathrm{Hz}$ and $154 \mathrm{dBc} / \mathrm{Hz}$ level at $1 \mathrm{~Hz}$ and $5 \mathrm{kHz}$ offset frequency, respectively, which results in 838 as $\mathrm{rms}$ timing jitter integrated from $1 \mathrm{~Hz}$ to $1 \mathrm{MHz}$. Note that the integrated jitter is concentrated in the $100 \mathrm{kHz}-1 \mathrm{MHz}$ range (819 as), whereas the jitter in $1 \mathrm{~Hz}-100 \mathrm{kHz}$ contributes only 179 as. The residual phase noise is ultimately limited by the phase noise of the free-running VCO outside the locking bandwidth, thus, it is important to use a highquality VCO to achieve sub-fs-level synchronization. Curve (iii) is the background noise of the optical phase detector, when only optical pulse train is applied to the phase detector without microwave signal. We believe that the background noise is mainly caused by the amplitudeto-phase conversion in the optical phase detector by imperfect coupling ratio ( $\pm 1 \%$ error) of the 50:50 coupler in the Sagnac-loop. Curve (iv) shows the in-loop phase error measured in the optical PLL. The measured out-ofloop phase noise is much higher than the projected in-loop phase noise because the PLL performance is limited by the additive phase noise in the optical phase detector, which cannot be suppressed by the loop gain.

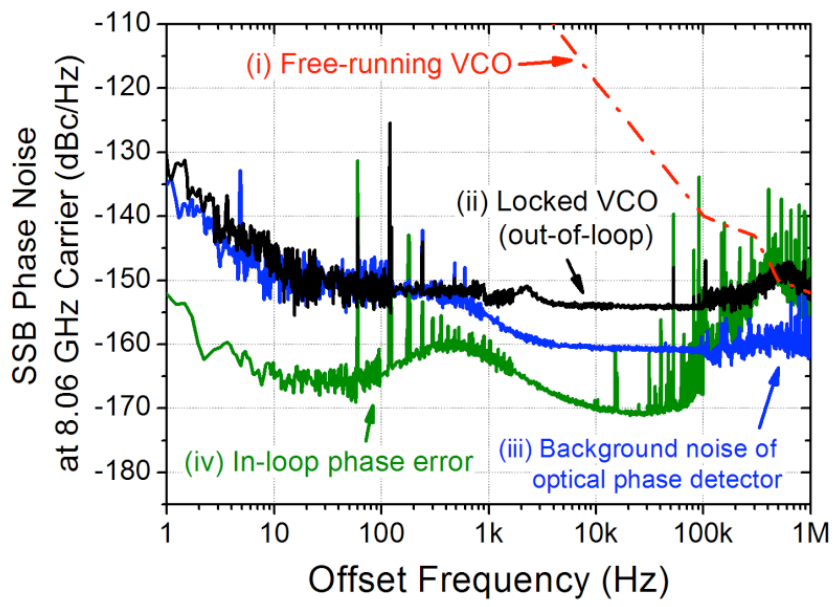

Fig. 3. (a) Signle-sideband (SSB) phase noise measurement results: (i) Phase noise of free-running VCO; (ii) Out-of-loop residual phase noise of VCO locked to the Er-fiber laser (integrated rms timing jitter $=838$ as $[1 \mathrm{~Hz}-1 \mathrm{MHz}]$ ); (iii) Background noise of optical phase detector when microwave signal is not applied; (iv) In-loop residual phase noise. 
Figure 4 shows the typical out-of-loop long-term phase drift measurement result between the optical pulse trains and the synchronized microwave signals sampled at 2 samples/s with a $100 \mathrm{~Hz}$ low-pass filter. For the 2-hour measurement (Fig. 4(a)), the integrated phase drift is 847 as (rms). The slow phase drift is mainly caused by the phase drift in the bias unit (originated from the temperature-dependent birefringence change in the quarter-wave plate) with a temperature coefficient of $\sim 20$ fs/K. Based on the measured relative timing drift, we also calculated the fractional frequency instability (in terms of overlapping Allan deviation). Starting from $1.5 \times 10^{-16}$ at 1 $\mathrm{s}$ averaging time, it reaches $4.0 \times 10^{-19}$ level at $1800 \mathrm{~s}$ averaging time (Fig. 4(b)) for a single system.

(a)

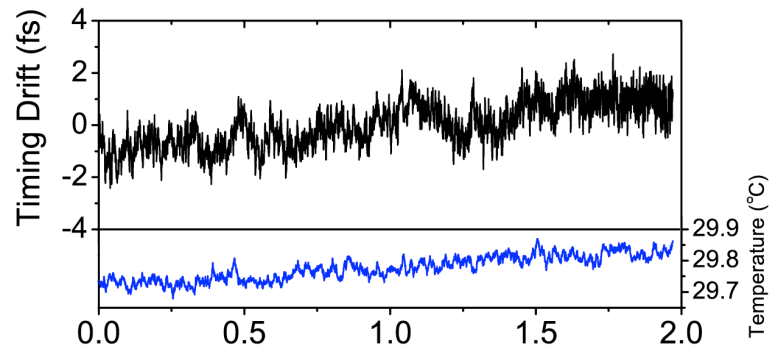

(b)

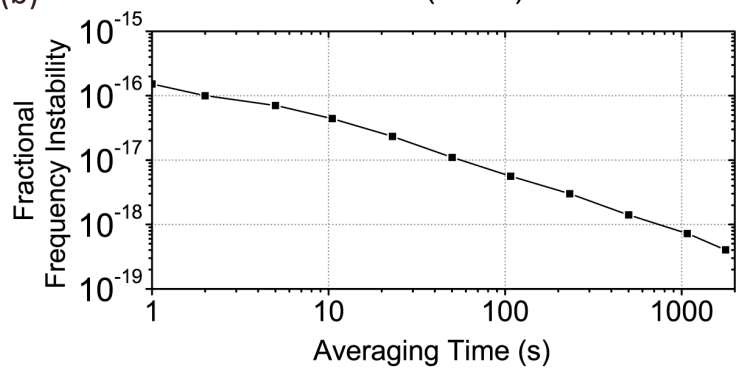

Fig. 4. Long-term residual phase drift measurement result between the optical pulse train and the synchronized microwave signal. (a) Measurement over 2 hours (847 as (rms) drift). (b) Fractional frequency instability (overlapping Allan deviation) based on the measured result in (a).

In summary, we have synchronized an $8.06-\mathrm{GHz}$ microwave oscillator with a $77.5-\mathrm{MHz}$ mode-locked $\mathrm{Er}^{-}$ fiber laser with residual phase noise of $-133 \mathrm{dBc} / \mathrm{Hz}(-154$ $\mathrm{dBc} / \mathrm{Hz})$ at $1 \mathrm{~Hz}(5 \mathrm{kHz})$ offset frequency, which results in 838 as integrated rms jitter [ $1 \mathrm{~Hz}-1 \mathrm{MHz}]$. Long-term phase drift is 847 as (rms) integrated for 2 hours. One of the advantages of this scheme is that there is no restriction on usable repetition rate of mode-locked lasers. Another advantage is that one can obtain stable highpower microwaves from mode-locked lasers without microwave amplification processes by using a high-power, high-quality VCO. One can further build highlysynchronized, ultralow-noise and remotely distributed microwave signal generators for driving accelerating cavities in free-electron lasers by combining the demonstrated PLL system with timing-stabilized fiber links [20]. The fiber-based optical-microwave phase detector itself can be also used for precise measurement of the phase noise of microwave sources or extracting ultrastable microwave signals from optical atomic clocks. As a future work we will measure the absolute phase noise of microwave signals using two independent ultralow jitter mode-locked Er-fiber lasers for the demonstration of ultralow phase noise microwave generators.

We thank Giuseppe Marra for fruitful discussions. This research was supported in part by the Technology Innovation Program funded by the Ministry of Knowledge and Economy (MKE) and the National Research Foundation (NRF) of Korea (2012R1A2A2A01005544).

\section{References}

1. For example, "PSI sapphire loaded cavity oscillator," http://psi.com.au/media/pdfs/SLCO-BCS Brochure.pdf.

2. For example, "OEwaves Opto-Electronic Oscillator," http://oewaves.com/products/item/84-compact-optoelectronic-oscillator-oeo.html.

3. T. M. Fortier, M. S. Kirchner, F. Quinlan, J. Taylor, J. C. Bergquist, T. Rosenband, N. Lemke, A. Ludlow, Y. Jiang, C. W. Oates, and S. A. Diddams, Nature Photon. 5, 425 (2011).

4. J. Millo, R. Boudot, M. Lours, P. Y. Bourgeois, A. N. Luiten, Y. Le Coq, Y. Kersalé, and G. Santarelli, Opt. Lett. 34, 3707 (2009).

5. W. Zhang, Z. Xu, M. Lours, R. Boudot, Y. Kersalé, G. Santarelli, and Y. Le Coq, Appl. Phys. Lett. 96, 211105 (2010).

6. F. Quinlan, T. M. Fortier, M. S. Kirchner, J. A. Taylor, M. J. Thorpe, N. Lemke, A. D. Ludlow, Y. Jiang, and S. A. Diddams, Opt. Lett. 36, 3260 (2011).

7. W. Zhang, Z. Xu, M. Lours, R. Boudot, Y. Kersalé, A. N. Luiten, Y. Le Coq, and G. Santarelli, IEEE Trans. Ultrason. Ferroelectr. Freq. Control 58, 900 (2011).

8. T. M. Fortier, C. W. Nelson, A. Hati, F. Quinlan, J. Taylor, H. Jiang, C. WS. Chou, N. Lemke, A. Ludlow, D. Howe, C. Oates, and S. A. Diddams, in Proceedings of IEEE Photonics Conference (IEEE, 2011), DOI:10.1109/ PHO.2011.6110874.

9. T. K. Kim, Y. Song, K. Jung, C. Kim, H. Kim, C. H. Nam, and J. Kim, Opt. Lett. 36, 4443 (2011).

10. Y. Song, C. Kim, K. Jung, H. Kim, and J. Kim, Opt. Express 19, 14518 (2011).

11. A. J. Benedick, J. G. Fujimoto, and F. X. Kärtner, Nature Photon. 6, 97 (2012).

12. J. Taylor, S. Datta, A. Hati, C. Nelson, F. Quinlan, A. Joshi, and S. Diddams, IEEE Photon. J. 3, 140 (2011).

13. B. Lorbeer, F. Ludwig, H. Schlarb, and A. Winter, in Proceedings of the Particle Accelerator Conference 2007 (2007), pp. 182-184.

14. K. Wu, P. P. Shum, S. Aditya, C. Ouyang, J. H. Wong, H. Q. Lam, and K. E. Lee, J. Lightw. Technol. 29, 3622 (2011).

15. A. Haboucha, W. Zhang, T. Li, M. Lours, A. N. Luiten, Y. Le Coq, and G. Santarelli, Opt. Lett. 36, 3654 (2011).

16. H. Jiang, J. Taylor, F. Quinlan, T. Fortier, and S. A. Diddams, IEEE Photon. J. 3, 1004 (2011).

17. J. Kim and F. X. Kärtner, Opt. Lett. 35, 2022 (2010).

18. J. Kim, F. X. Kärtner, and M. H. Perrott, Opt. Lett. 29, 2076 (2004).

19. M. L. Dennis, I. N. Duling III, and W. K. Burns, Electron. Lett. 32, 547 (1996).

20. J. Kim, J. Cox, J. Chen, and F. X. Kärtner, Nature Photon. 2, 733 (2008). 


\section{Full references}

1. For example, "PSI sapphire loaded cavity oscillator," http://psi.com.au/media/pdfs/SLCO-BCS Brochure.pdf.

2. For example, "OEwaves Opto-Electronic Oscillator," http://oewaves.com/products/item/84-compact-optoelectronic-oscillator-oeo.html.

3. T. M. Fortier, M. S. Kirchner, F. Quinlan, J. Taylor, J. C. Bergquist, T. Rosenband, N. Lemke, A. Ludlow, Y. Jiang, C. W. Oates, and S. A. Diddams, "Generation of ultrastable microwaves via optical frequency division," Nature Photon. 5, 425-429 (2011).

4. J. Millo, R. Boudot, M. Lours, P. Y. Bourgeois, A. N. Luiten, Y. Le Coq, Y. Kersalé, and G. Santarelli, "Ultra-low-noise microwave extraction from fiber-based optical frequency comb," Opt. Lett. 34, 3707-3709 (2009).

5. W. Zhang, Z. Xu, M. Lours, R. Boudot, Y. Kersalé, G. Santarelli, and Y. Le Coq, "Sub-100 attoseconds stability optics-to-microwave synchronization,” Appl. Phys. Lett. 96, 211105 (2010)

6. F. Quinlan, T. M. Fortier, M. S. Kirchner, J. A. Taylor, M. J. Thorpe, N. Lemke, A. D. Ludlow, Y. Jiang, and S. A. Diddams, "Ultralow phase noise microwave generation with an Er:fiber-based optical frequency divider," Opt. Lett. 36, 3260-3262 (2011).

7. W. Zhang, Z. Xu, M. Lours, R. Boudot, Y. Kersalé, A. N. Luiten, Y. Le Coq, and G. Santarelli, "Advanced Noise Reduction Techniques for Ultra-Low Phase Noise Opticalto-Microwave Division With Femtosecond Fiber Combs," IEEE Trans. Ultrason. Ferroelectr. Freq. Control 58, 900908 (2011).

8. T. M. Fortier, C. W. Nelson, A. Hati, F. Quinlan, J. Taylor, H. Jiang, C. W. Chou, N. Lemke, A. Ludlow, D. Howe, C. Oates, and S. A. Diddams, "A hybrid $10 \mathrm{GHz}$ photonicmicrowave oscillator with sub-femtosecond absolute timing jitter," in Proceedings of IEEE Photonics Conference (IEEE, 2011), DOI:10.1109/ PHO.2011.6110874.

9. T. K. Kim, Y. Song, K. Jung, C. Kim, H. Kim, C. H. Nam, and J. Kim, "Sub-100-as timing jitter optical pulse trains from mode-locked Er-fiber lasers," Opt. Lett. 36, 44434445 (2011).

10. Y. Song, C. Kim, K. Jung, H. Kim, and J. Kim, "Timing jitter optimization of mode-locked Yb-fiber lasers toward the attosecond regime," Opt. Express 19, 14518-14525 (2011).

11. A. J. Benedick, J. G. Fujimoto, and F. X. Kärtner, "Optical flywheels with attosecond jitter," Nature Photon. 6, 97-100 (2012).

12. J. Taylor, S. Datta, A. Hati, C. Nelson, F. Quinlan, A. Joshi, and S. Diddams, "Characterization of Power-toPhase Conversion in High-Speed P-I-N Photodiodes," IEEE Photon. J. 3, 140-151 (2011).

13. B. Lorbeer, F. Ludwig, H. Schlarb, and A. Winter, "Noise and drift characterization of direct laser to $\mathrm{rf}$ conversion scheme for the laser based synchronization system for FLASH at DESY," in Proceedings of the Particle Accelerator Conference 2007 (2007), pp. 182-184.

14. K. Wu, P. P. Shum, S. Aditya, C. Ouyang, J. H. Wong, H. Q. Lam, and K. E. Lee, "Characterization of the Excess Noise Conversion From Optical Relative Intensity Noise in the Photodetection of Mode-Locked Lasers for Microwave Signal Synthesis," J. Lightw. Technol. 29, 3622-3631 (2011).

15. A. Haboucha, W. Zhang, T. Li, M. Lours, A. N. Luiten, Y. Le Coq, and G. Santarelli, "Optical-fiber pulse rate multiplier for ultralow phase-noise signal generation," Opt. Lett. 36, 3654-3656 (2011).
16. H. Jiang, J. Taylor, F. Quinlan, T. Fortier, and S. A. Diddams, "Noise Floor Reduction of an Er:Fiber LaserBased Photonic Microwave Generator," IEEE Photon. J. 3, 1004-1012 (2011).

17. J. Kim and F. X. Kärtner, "Microwave signal extraction from femtosecond mode-locked lasers with attosecond relative timing drift," Opt. Lett. 35, 2022-2024 (2010).

18. J. Kim, F. X. Kärtner, and M. H. Perrott, "Femtosecond synchronization of radio frequency signals with optical pulse trains," Opt. Lett. 29, 2076-2078 (2004).

19. M. L. Dennis, I. N. Duling III, and W. K. Burns, "Inherently bias drift free amplitude modulator," Electron. Lett. 32, 547-548 (1996).

20. J. Kim, J. Cox, J. Chen, and F. X. Kärtner, "Drift-free femtosecond timing synchronization of remote optical and microwave sources," Nature Photon. 2, 733-736 (2008). 